\title{
Immunohistochemical expression of geminin in colorectal cancer: Implication of prognostic significance
}

\author{
KEISUKE NISHIHARA ${ }^{1,2}$, KOHEI SHOMORI ${ }^{1}$, TAKAYUKI TAMURA ${ }^{1}$, \\ SHINJI FUJIOKA ${ }^{1}$, TOSHIHIDE OGAWA ${ }^{2}$ and HISAO ITO ${ }^{1}$ \\ ${ }^{1}$ Division of Organ Pathology, Department of Microbiology and Pathology, \\ ${ }^{2}$ Division of Radiology, Department of Pathophysiological and Therapeutic Science, \\ Tottori University Faculty of Medicine, 86 Nishi-cho, Yonago, Tottori 683-8503, Japan
}

Received November 6, 2008; Accepted January 12, 2009

DOI: 10.3892/or_00000340

\begin{abstract}
DNA should be duplicated precisely once per cell cycle to maintain genome integrity. After DNA replication, geminin binding to $\mathrm{Cdt} 1$ inhibits uploading of the minichromosome maintenance (MCM) complex as DNA helicase onto chromatin and prevents DNA re-replication in the same cell cycle. Expression of geminin indicates poor prognosis in some malignancies, such as breast and renal cell carcinoma. We evaluated the expression of geminin to clarify its pathobiological and prognostic significance in colorectal cancer, compared with expression of MCM7 and Ki-67. We performed Western blot analyses of 5 human colorectal cancer cell lines and immunohistochemistry on 191 surgically removed specimens of Dukes' B and C stage colorectal cancer. Doublelabeling immunofluorescence was also carried out to identify co-expression of geminin, MCM7 and Ki-67. Geminin proteins were detected in all the 5 cell lines examined. Geminin, MCM7 and $\mathrm{Ki}-67$ were co-expressed and cells that stained only for geminin were not detected. Mean labeling indices (LIs) for geminin, MCM7 and Ki-67 were 26.3, 58.2 and 40.8\%, respectively. Patients with high geminin LIs had significantly unfavorable prognosis in stage II and III colorectal cancer $(\mathrm{P}=0.04)$. Patients with a tumor with a higher proliferating nature (i.e. high LIs for three markers) showed significantly unfavorable prognosis in multivariate Cox analysis. Our results indicate that assessment of geminin, MCM7 and Ki-67 may be useful for predicting prognosis in patients with colorectal cancer.
\end{abstract}

Correspondence to: Dr Kohei Shomori, Division of Organ Pathology, Department of Microbiology and Pathology, Tottori University Faculty of Medicine, 86, Nishi-cho, Yonago, Tottori 683-8503, Japan

E-mail: shomori@med.tottori-u.ac.jp

Key words: geminin, minichromosome maintenance 7, Ki-67, prognostic factor, colorectal cancer

\section{Introduction}

Colorectal cancer is one of the most common cancers worldwide, but the current TNM classification system does not necessarily accurately predict the prognosis of individual patients $(1,2)$. Therefore, new molecular markers should be identified to predict prognosis in patients with colorectal cancer.

One of the crucial points for genomic stability is DNA replication, since this process should ensure that the entire genome is precisely duplicated once per cell cycle. DNA replication licensing involves the stepwise assembly of initiator proteins, including $\mathrm{Cdt} 1$ and minichromosome maintenance (MCM) protein complex consisting of MCM2-7, on origins of replication (3). The activated MCM complex appears to play a key role in the DNA unwinding step, by acting as a DNA helicase (3). After DNA replication is initiated during the cell cycle, geminin binds to Cdt1 and inhibits re-uploading of the MCM complex onto chromatin, thus preventing DNA re-replication in the same cell cycle (4).

Although it has been suggested that geminin possesses possible tumor suppressive functions $(5,6)$, immunohistochemistry and immunoblotting have revealed that geminin is absent in organs or tissues with minimal proliferating activity, such as normal liver and is specifically expressed in proliferating lymphocytes, male germ cells and various epithelial cells, including those in the skin, uterine cervix and colon (6-8). Moreover, previous studies have demonstrated that expression of geminin increases with tumor grade (9-13), which leads to poor prognosis in a variety of cancer patients, such as those with breast and renal cell carcinomas $(14,15)$.

$\mathrm{Ki}-67$ is a cell cycle entry marker that is expressed during all the phases of the cell cycle except for G0 (16) (Fig. 1). Previous studies have shown that MCM proteins are expressed in both rapidly and slowly cycling populations, such as primary oocytes and premenopausal breast cells $(8,17)$. Geminin is expressed during the $\mathrm{S}, \mathrm{G} 2$ and $\mathrm{M}$ phases but not in G0 and G1 phases (18).

Overexpression of geminin has been reported in colorectal cancer, but its prognostic significance has not been established (9). We have also previously reported the expression of 
MCM7 and Ki-67 and a high labeling index (LI) for MCM7 is an independent prognostic factor in colorectal cancer (19). Here, we focused on the expression of geminin in human colorectal cancer. Furthermore, we assessed the prognostic significance of the nature of the tumor as estimated by expression of MCM7, Ki-67 and geminin.

\section{Materials and methods}

Cell culture and Western blot analysis. The human colon carcinoma cell lines Colo201, Colo320, DLD-1, LoVo and WiDr were routinely cultured in RPMI-1640 supplemented with $10 \%$ fetal bovine serum at $37^{\circ} \mathrm{C}$ in a $5 \% \mathrm{CO}_{2}$-containing atmosphere.

Western blot analyses were processed using protocol, as described previously (19). The membrane with resulting protein samples were incubated with primary antibodies at the following dilutions: rabbit anti-geminin antibody (1:1000; Santa Cruz Biotechnology, Santa Cruz, CA); and mouse antiB-actin (1:2000; Sigma, St. Louis, MO). Next, the membranes were incubated with horseradish peroxidase-conjugated anti-rabbit IgG (1:2000; MBL, Nagoya, Japan) and anti-mouse $\operatorname{IgG}(1: 2000 ; \mathrm{MBL})$.

Surgical specimens. A total of 191 colorectal carcinoma specimens diagnosed as Dukes' B or C stage were selected from the files of Yonago Medical Center Hospital (Yonago, Japan). All the patients underwent radical en bloc resection between April 2000 and December 2005. No patients received preoperative chemotherapy and/or radiotherapy. Among these, 132 carcinomas were located in the colon and 59 in rectum. Ninety-five carcinomas were classified as Dukes' B stage and 96 as $\mathrm{C}$ stage. There were 108 male and 83 female patients. The median age was 68 years, with a range of 33-94 years. The mean follow-up period was 35.5 months, with a maximum of 89.9 months.

After routine processing, the surgical specimens were fixed in $10 \%$ buffered formalin and embedded in paraffin wax. All tumors were routinely stained with hematoxylin and eosin. Histological diagnosis was made according to the TNM staging system $(1,2)$ and the 7 th edition of the General Rules for Clinical and Pathological Studies on Cancer of the Colon, Rectum and Anus (20). The study protocol was approved by the Institutional Review Boards of Tottori University Faculty of Medicine.

Immunohistochemistry. Immunohistochemistry was performed using a standard streptavidin-biotin-peroxidase complex protocol, as described previously (19). The sections were incubated with the following antibodies: rabbit anti-geminin antibody (1:100); mouse anti-MCM7 antibody (1:100; Santa Cruz Biotechnology); and mouse anti-Ki-67 antibody (1:50; Dako, Glostrup, Denmark).

Quantitative analysis was undertaken by two of the authors, who were unaware of the clinicopathological variables. Counts were performed in high-magnification fields from the most intensely stained areas using the Flovel Image Filing System FlvFs (Flovel Inc., Tachikawa, Japan). Cells were identified as positive if there was any nuclear staining present and any stromal or inflammatory cells in the field were excluded. An
Table I. The correlation between the geminin labeling index and clinicopathological profiles.

\begin{tabular}{|c|c|c|c|c|c|}
\hline & & & Geminin & & \\
\hline & $\mathrm{N}$ & Mean LI (\%) & $\mathrm{LI}>25 \%$ & $\mathrm{LI} \leq 25 \%$ & P-value \\
\hline Age & & & & & 0.06 \\
\hline$\geq 68$ & 98 & 25.5 & 53 & 45 & \\
\hline$<68$ & 93 & 27.3 & 63 & 30 & \\
\hline Gender & & & & & 0.86 \\
\hline Female & 83 & 26.9 & 51 & 32 & \\
\hline Male & 108 & 25.9 & 65 & 43 & \\
\hline Tumor & & & & & \\
\hline location & & & & & 0.79 \\
\hline Colon & 132 & 26.4 & 81 & 51 & \\
\hline Rectum & 59 & 26.2 & 35 & 24 & \\
\hline $\begin{array}{l}\text { Histological } \\
\text { differentiation }\end{array}$ & & & & & 0.04 \\
\hline Well & 84 & 27.5 & 57 & 27 & \\
\hline Moderately & 92 & 25.9 & 54 & 38 & \\
\hline Poorly & 15 & 22.1 & 5 & 10 & \\
\hline Dukes' stage & & & & & 0.70 \\
\hline B & 95 & 26.7 & 59 & 36 & \\
\hline $\mathrm{C}$ & 96 & 26.0 & 57 & 39 & \\
\hline $\mathrm{T}$ stage & & & & & 0.63 \\
\hline $\mathrm{T} 1+2$ & 11 & 25.3 & 7 & 4 & \\
\hline $\mathrm{T} 3$ & 155 & 26.7 & 96 & 59 & \\
\hline $\mathrm{T} 4$ & 25 & 24.6 & 13 & 12 & \\
\hline $\mathrm{N}$ stage & & & & & 0.35 \\
\hline NO & 95 & 26.7 & 59 & 36 & \\
\hline $\mathrm{N} 1$ & 69 & 26.9 & 44 & 25 & \\
\hline $\mathrm{N} 2$ & 27 & 23.5 & 13 & 14 & \\
\hline $\begin{array}{l}\text { Distant } \\
\text { metastasis }\end{array}$ & & & & & 0.80 \\
\hline M0 & 157 & 26.5 & 96 & 61 & \\
\hline M1 & 34 & 25.7 & 20 & 14 & \\
\hline UICC stage & & & & & 0.97 \\
\hline II & 93 & 26.7 & 57 & 36 & \\
\hline III & 64 & 26.2 & 39 & 25 & \\
\hline IV & 34 & 25.7 & 20 & 14 & \\
\hline MCM7 LI & & & & & $<0.01$ \\
\hline$>58.0$ & 101 & 28.4 & 71 & 28 & \\
\hline$\leq 58.0$ & 90 & 24.0 & 45 & 46 & \\
\hline Ki-67 LI & & & & & $<0.01$ \\
\hline$>40.0$ & 103 & 28.6 & 72 & 31 & \\
\hline$\leq 40.0$ & 88 & 23.7 & 44 & 43 & \\
\hline
\end{tabular}

LI, labeling index.

average of 800 nuclei was counted for each case. LI was calculated by dividing the number of positive cells by the total number of cells counted.

Double-labeling immunofluorescence. Paraffin-embedded tissue sections were processed for double-labeling immunofluorescence protocol, as described previously (19). The 


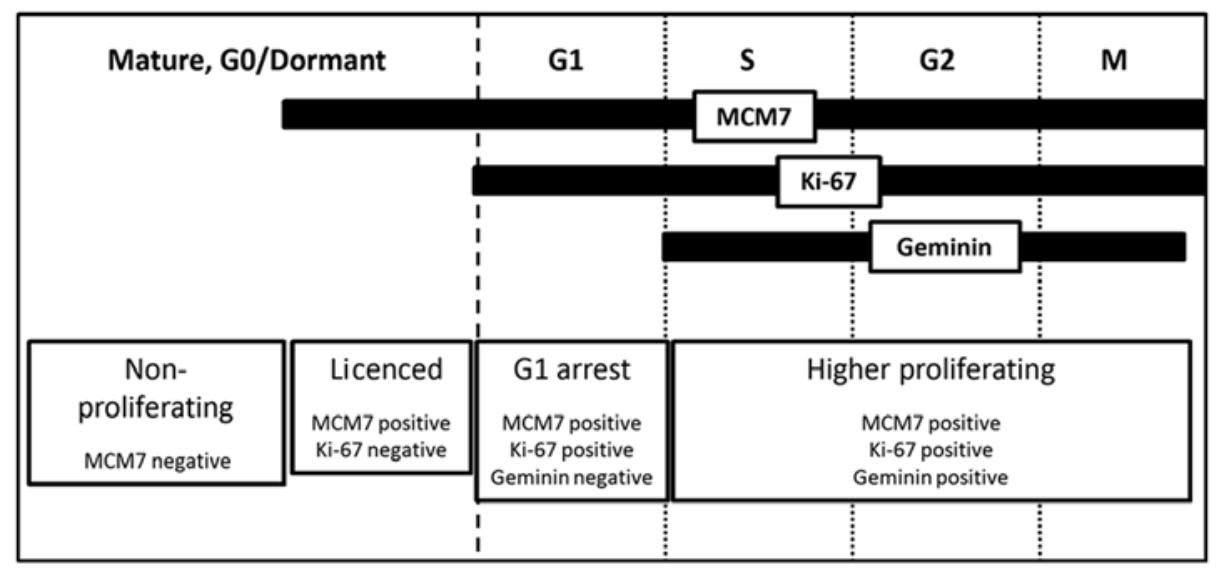

Figure 1. Cell cycle-specific expression of MCM7, Ki-67 and geminin. MCM7 is expressed in cells that possessed proliferation potential during all phases of the cell cycle. Ki-67 is expressed during all phases of the cell cycle except for G0. Geminin is expressed during S, G2 and M phases. Therefore, we classify four cell cycle kinetics patterns according to expression of geminin, MCM7 and Ki-67. MCM7 high, Ki-67 high and geminin high are classified when the LIs of immunoreactive tumor cells were $>58,40$ and $25 \%$, respectively.

sections were incubated with a mixture of anti-geminin antibody (1:100) and anti-MCM7 antibody (1:100) and a mixture of anti-geminin antibody (1:100) and anti-Ki-67 antibody (1:100). Incubation with R-phycoerythrin-conjugated goat anti-rabbit IgG (1:200; Molecular Probes Inc., Eugene, OR, USA) was carried out, followed by incubation with Alexa Fluor 488-conjugated rabbit anti-mouse IgG (1:200; Molecular Probes Inc.) and mounted. The sections were observed using a fluorescence microscope (Eclipse E800; Nikon, Tokyo, Japan).

Statistical analysis. Correlations between the LIs for geminin, MCM7 and Ki-67 were analyzed using Pearson's correlation coefficient test. Correlations between the LIs and clinicopathological variables were analyzed using analyses of two values with the Mann-Whitney $U$ test and more than two values with the Kraskal-Wallis rank test. Kaplan-Meier cumulative survival curves were constructed for the LIs (21). A log-rank test was used to assess the statistical significance of these curves. Univariate and multivariate Cox regression models for survival were used to evaluate the contributions of various factors (22). $\mathrm{P}<0.05$ was considered to indicate statistical significance. The analyses were carried out using Statcel ver. 2 for Windows (OMS Publishing Inc., Saitama, Japan) and Dr. SPSS II for Windows (SPSS Inc., Tokyo, Japan).

\section{Results}

Geminin protein expression in human colorectal cancer cell lines. Initially, we examined the expression of geminin in five human colorectal cancer cell lines by Western blotting. As shown in Fig. 2, geminin proteins were detected at various levels in all the five colorectal carcinoma cell lines as clear single bands of $30 \mathrm{kDa}$, indicating the specificity of the antibody used.

Immunohistochemistry and double-labeling immunofluorescence. Immunoreactivity for geminin, MCM7 and Ki-67 was noted exclusively in the nucleus of tumor cells (Fig. 3A-D).

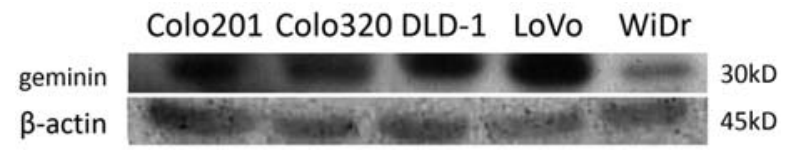

Figure 2. Western blot analysis for geminin expression in the five human colorectal cancer cell lines. Geminin proteins are expressed in all the five cell lines with specific bands of $30 \mathrm{kD}$.

Geminin was also immnoreactive in the perichromosomal cytoplasm of mitotic cells. Positive cells were observed in the basal third of crypts that corresponded to the proliferative zone and in the germ center of lymphoid follicles in non-neoplastic mucosa (Fig. 3E-G).

Double-labeling confocal immunofluorescence microscopy was conducted to identify co-expression of geminin and MCM7 and of geminin and Ki-67 (Fig. 3H-M). All gemininpositive cells (red) also expressed MCM7 (green) (Fig. 3J), which produced a yellow signal when the images were merged. Similarly, all geminin-positive cells (red) expressed Ki-67 (green) (Fig. 3M). Geminin-only-positive cells (red fluorescent signal alone) were not detected after merging.

Geminin, MCM7 and Ki-67 immunoreactive tumor cells were more frequently observed in the marginal area at the invasive front than in the center of the tumor. Therefore, we mainly evaluated the marginal areas. The mean LIs of geminin, MCM7 and Ki-67 were 26.3 $\pm 8.5,58.2 \pm 9.2$ and $40.8 \pm 10.3 \%$, respectively. There was a significant positive correlation in LI between geminin and MCM7 $(r=0.20$, $\mathrm{P}<0.01)$, as well as geminin and $\mathrm{Ki}-67(\mathrm{r}=0.26, \mathrm{P}<0.01)$.

Correlations between geminin expression and clinicopathological profile. We evaluated the correlations between LIs for geminin and clinicopathological profiles (Table I). We chose the cut-off value using the 'minimum P-value approach' (23); that is, geminin LI of 25\%, MCM7 LI of 58\% and Ki-67 LI of $40 \%$, the value being almost equal to the mean LIs. High LIs for geminin, MCM7, and Ki-67 were noted in 116, 101 and 103 carcinomas, respectively. 

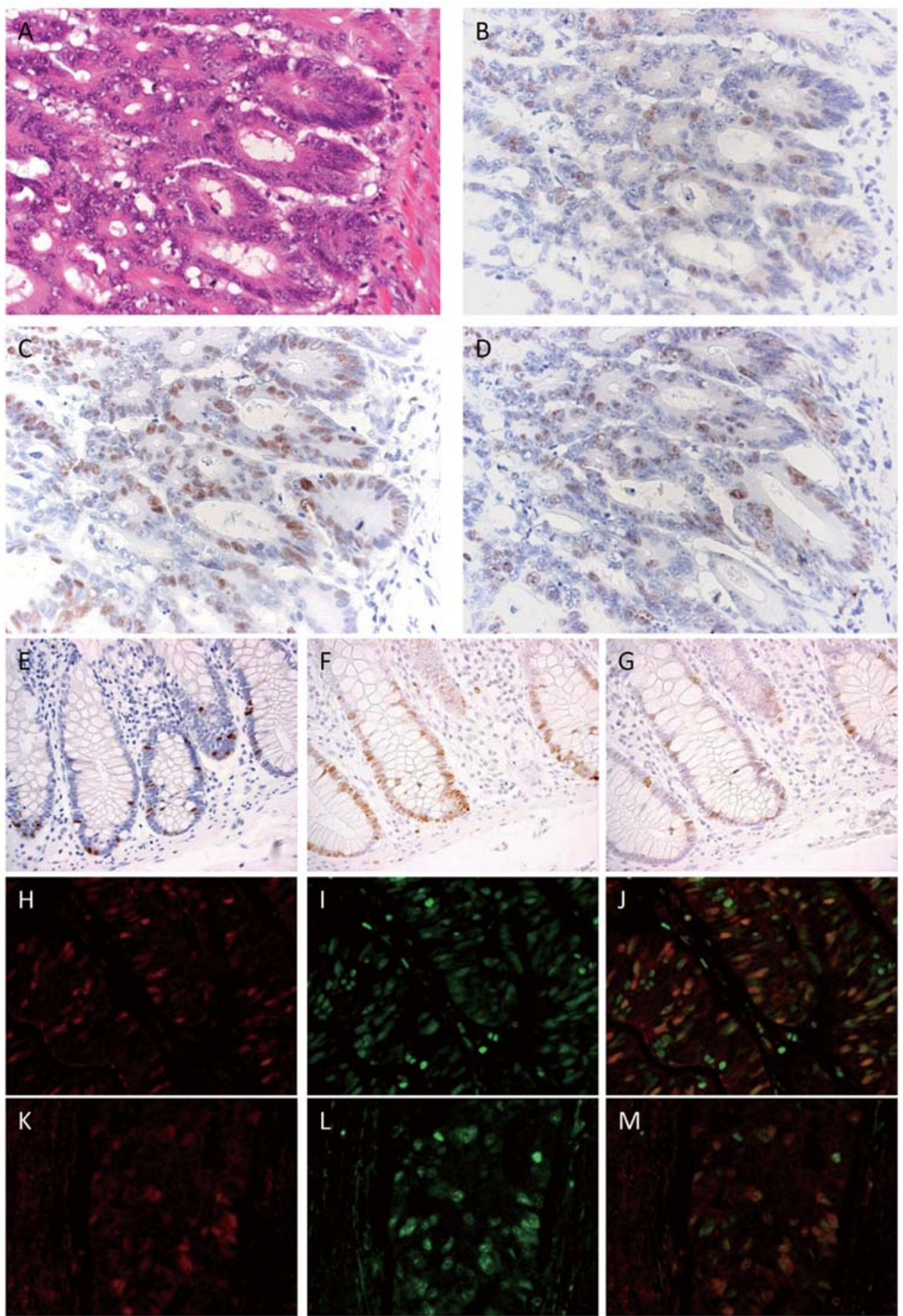

Figure 3. Immunohistochemistry for geminin, MCM7 and Ki-67 and double-labeling immunofluorescence for geminin and MCM7 or Ki-67 in human colorectal cancer specimens. Serial sections stained with hematoxylin and eosin (A) and immunohistochemically stained for geminin (B), MCM7 (C) and Ki-67 (D) in tumor and for geminin (E), MCM7 (F) and Ki-67 (G) in non-tumoral epithelia are shown. Double-labeling immunofluorescence for geminin (H) and MCM7 (I), and geminin (K) and Ki-67 (L) were performed. All tumor cells positive for geminin were found to co-express MCM7 (J) or Ki-67 (M) in the merged image (yellow).

LI for geminin was significantly higher in the welldifferentiated than in the poorly differentiated tumors $(\mathrm{P}<0.05)$. The LI for geminin was significantly correlated with MCM7 high LI (>58\%) and Ki-67 high LI (>40\%) $(\mathrm{P}<0.01)$, but not with age, gender, tumor location, Dukes' stage, $\mathrm{T}$ stage, $\mathrm{N}$ stage, distant metastasis and UICC stage.

Analysis of prognosis. We examined the cumulative survival of two groups of patients with high and low LIs using the
Kaplan-Meier method and log-rank test. A significantly poorer prognosis was found in the 96 patients with a higher geminin LI compared to the 61 with a lower geminin LI in stage II and III ( $\mathrm{P}=0.04 ;$ Fig. $4 \mathrm{~A})$. The patients with a high geminin LI showed poorer survival, even in stage II ( $\mathrm{P}=0.05$; Fig. 4B).

Colorectal cancer with higher LIs of MCM7, Ki-67 and geminin might be characterized as higher proliferating tumors, which were noted in 52 of our cases. The 43 patients with the higher proliferating tumors showed significantly poorer 
A
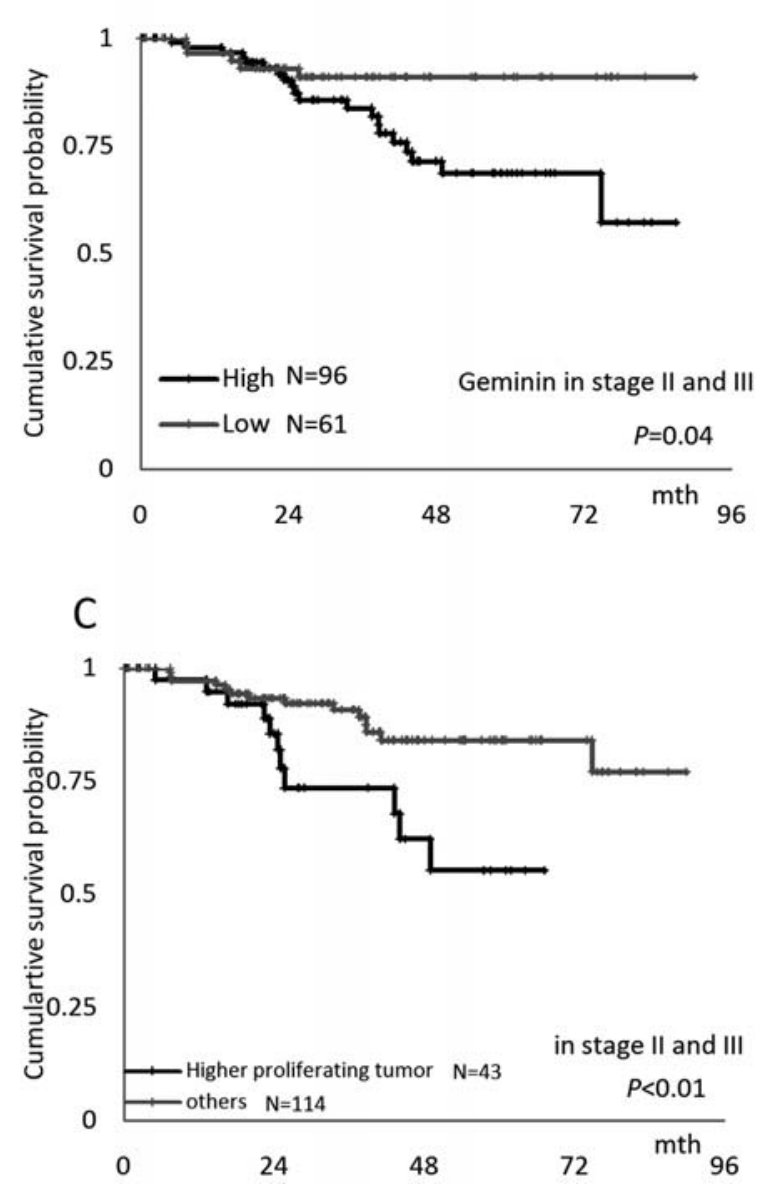

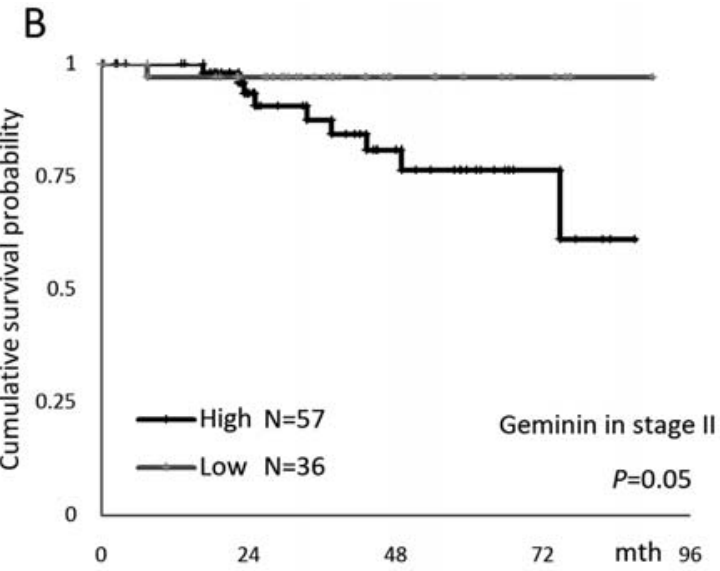

D

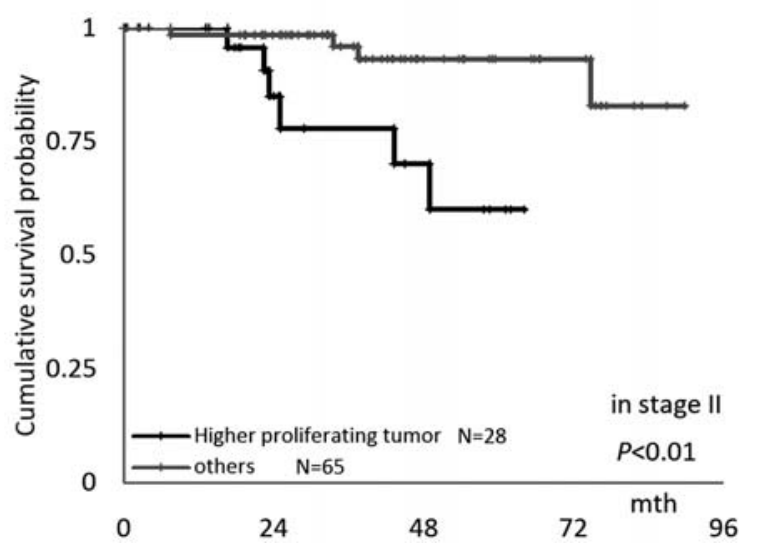

Figure 4. Kaplan-Meier curves showing cumulative overall survival rates according to the markers. Geminin LI of $25 \%$ was chosen as a cut-off value. The cumulative survival of the 96 patients with a high geminin LI is significantly poorer than that of the 61 patients with a low LI in stage II and III ( $\mathrm{P}=0.04)(\mathrm{A})$. Patients with high geminin LI in stage II show poorer survival ( $\mathrm{P}=0.05)(\mathrm{B})$. Patients with higher proliferating tumors, high MCM7 LI ( $>58 \%)$, high Ki-67 LI $(>40 \%)$ and high geminin LI $(>25 \%)$ show a significantly more unfavorable prognosis in stage II-III $(\mathrm{P}<0.01)(\mathrm{C})$ and even in stage II $(\mathrm{P}<0.01)(\mathrm{D})$.

prognosis than the other 114 patients in stage II and III $(\mathrm{P}<0.01$; Fig. 4C). Similarly, the 28 patients with the higher proliferating tumors showed significantly poorer prognosis than the others, even in stage II $(\mathrm{P}<0.01 ;$ Fig. 4D).

We performed univariate Cox regression analyses to evaluate the contributions of potential prognostic markers to overall survival. Overall survival was significantly correlated with high LI for geminin [relative risk $(\mathrm{RR})=2.15 ; \mathrm{P}=0.03$ ] and high LI for MCM7 (RR=2.30; $\mathrm{P}=0.01$ ) (Table II). Patients with higher proliferating tumors were also significantly correlated with overall survival rate $(\mathrm{RR}=2.35 ; \mathrm{P}<0.01)$.

A multivariate Cox regression analysis was undertaken to determine whether the markers examined offered prognostic information compared with age, gender, tumor location, histological differentiation, $\mathrm{T}$ stage, $\mathrm{N}$ stage, $\mathrm{M}$ status and UICC stage. Significant and independent prognostic factors in this analysis included UICC stage, which showed the highest $\mathrm{RR}$ value, followed by $\mathrm{T}$ stage and the higher proliferating tumors $(\mathrm{RR}=2.25 ; \mathrm{P}<0.01)$ (Table II).

\section{Discussion}

This study clearly demonstrated the prognostic significance of geminin and tumor pattern from the cell cycle point of view,
Table II. Univariate and multivariate Cox regression analysis of the contribution of various parameters to the overall survival.

\begin{tabular}{lcccc}
\hline & & RR & $95 \%$ CI & P-value \\
\hline Univariate analysis & & & & \\
Geminin & $>25 \%$ & 2.15 & $1.09-4.22$ & 0.03 \\
MCM7 & $>58 \%$ & 2.30 & $1.24-4.25$ & 0.01 \\
Ki-67 & $>40 \%$ & 1.08 & $0.61-1.92$ & 0.80 \\
$\begin{array}{l}\text { Higher proliferating } \\
\text { tumor }\end{array}$ & & & & \\
& vs. others & 2.35 & $1.30-4.24$ & $<0.01$ \\
Multivariate analysis & & & & \\
Higher proliferating & & & & \\
tumor & vs. others & 2.25 & $1.23-4.11$ & $<0.01$ \\
UICC Stage & vs. II & & & \\
$\quad$ III & & 2.46 & $1.08-5.57$ & 0.03 \\
IV & & 9.09 & $4.24-19.52$ & $<0.01$ \\
T stage & vs. T1+2 & & & \\
T3 & & 1.48 & $0.43-5.11$ & 0.53 \\
T4 & & 3.75 & $1.01-13.90$ & 0.05 \\
\hline
\end{tabular}

$\mathrm{RR}$, relative risk and $\mathrm{CI}$, confidence interval. 
using the immunohistochemical expression of geminin, MCM7 and Ki-67 in human colorectal cancer.

The LI for geminin was significantly higher in the welldifferentiated than in the poorly differentiated tumors, while poor survival was shown in patients with high LI for geminin. It is believed that the patients with the histological diagnosis of well-differentiated tumor usually have good prognosis. We speculate that the poorly differentiated tumors may have powerful potential to invade rather than to proliferate. Moreover, patients with high LIs for geminin showed unfavorable prognosis in colorectal cancer, although the LI for geminin was not correlated with any of the current TNM classification, such as $\mathrm{T}$ stage, $\mathrm{N}$ status and distant metastasis. We speculate that the LI for geminin may give information of proliferating tumors that are in S-G2-M phase, while the current TNM classification may be assessed by the tumor potential to invade and metastasize, but not by proliferating potential.

Quaglia et al have investigated co-expression of MCM2, $\mathrm{Ki}-67$ and geminin in hepatocellular carcinoma and precursor lesions in a small series of cirrhotic livers (24), in which nodules were classified into four patterns: negative for MCM2, Ki-67 and geminin; positive for MCM2 but negative for Ki-67 and geminin; positive for MCM2 and Ki-67 but only a few geminin-positive cells; and positive for all these markers. The pathobiological significance of these patterns, however, was not determined.

From the cell kinetics point of view, colorectal carcinoma consists of four types of tumor cells, as shown in Fig. 1. First, all MCM7-, Ki-67- and geminin-positive tumor cells are considered to be in S, G2 and M phase and possess high proliferation potential. Second, MCM7- and Ki-67-positive, geminin-negative cells are in G1 phase and may show proliferating activity. Third, MCM7-positive, Ki-67- and geminin-negative tumor cells are in G0/dormant phase. These tumor cells have been characterized as liscenced nonproliferating oocytes or cancer stem cells (19). Tumor cells that are negative for all three molecules, which correspond to MCM7-negative cells, might be non-proliferating cells. Mean LI for MCM7 was $58.2 \%$ in the present study, which implies that approximately half of the colorectal carcinoma cells remain in non-proliferating status. We focused our attention on colorectal carcinomas with higher LIs for MCM7, Ki-67 and geminin, because the carcinomas are expected to possess higher proliferating activity. In fact, the 43 patients with carcinoma that had a seemingly higher proliferating activity showed unfavorable prognosis compared with those in stage II and III. Moreover, univariate and multivariate analyses obviously showed that the simultaneously higher LIs for these three molecules is a significant and independent prognostic factor.

The patients with a high LI for geminin or MCM7 also showed poorer prognosis than those with other colorectal cancers. Patients with higher proliferating tumors, with higher LIs for geminin, MCM7 and Ki-67, had significantly poorer prognosis. Thus, simultaneously higher LIs for geminin, MCM7 and Ki-67 were a more powerful predictor of prognosis than a high LI for geminin or MCM7 alone, as estimated by RR and P-values. Moreover, both geminin and MCM7 LIs were omitted from the final Cox analysis, as they had no influence when this categorization was included. It might be necessary for the assessment of prognosis to have both the tumor cells in S, G2 and M phase and high proliferating potential. Therefore, we consider that the assessment of geminin, MCM7 and Ki-67 may be more useful than geminin or MCM7 alone for predicting prognosis in patients with colorectal cancer.

There are some limitations in the present study. We did not analyze the relationship between LI and prognosis in stage IV cancer, which showed obviously poorer prognosis. Moreover, we did not examine early or stage I cancer, which usually has a favorable prognosis, regardless of tumor pattern from the cell cycle point of view, using the LI for geminin, MCM7 and Ki-67.

In conclusion, our results indicate that geminin is overexpressed in colorectal cancer. Assessment of geminin, MCM7 and Ki-67 may be useful for predicting prognosis in patients with stage II or III colorectal cancer.

\section{Acknowledgements}

We thank Dr K. Yamaga and Dr K. Kidani in our group for their helpful discussions and research suggestions. We also thank Mr. N. Itaki, Ms. C. Yamasaki and Ms. M. Iwatani for excellent technical assistance and Dr H. Kawaguchi and Ms. M. Hashimoto for assistance in collecting patient information. This study received support from programs of the Encourage Fund 2006 and 2007 of Tottori University Graduate School of Medical Science. This study was also supported, in part, by a Grant-in-Aid for Scientific Research from the Ministry of Education, Culture, Sports, Science and Technology, Japan (Grant No. 14370069).

\section{References}

1. Mori T: New general rules on cancer of the colon, rectum and anus. Geka 9: 1030-1036, 2006. (In Japanese)

2. O'Connell JB, Maggard MA and Ko CY: Colon cancer survival rates with the new American Joint Committee on Cancer Sixth Edition Staging. J Natl Cancer Inst 96: 1420-1425, 2004.

3. Nishitani $\mathrm{H}$ and Lygerou $\mathrm{Z}$ : Control of DNA replication licensing in a cell cycle. Genes Cells 7: 523-534, 2002.

4. Pitulescu M, Kessel M and Luo L: The regulation of embryonic patterning and DNA replication by geminin. Cell Mol Life Sci 62: 1425-1433, 2005.

5. Lygerou Z and Nurse P: Cell cycle. License withheld - geminin blocks DNA replication. Science 290: 2271-2273, 2000.

6. Wohlschlegel JA, Kutok JL, Weng AP and Dutta A: Expression of geminin as a marker of cell proliferation in normal tissues and malignancies. Am J Pathol 161: 267-273, 2002.

7. Hansel DE, Rahman A, Hidalgo M, et al: Identification of novel cellular targets in biliary tract cancers using global gene expression technology. Am J Pathol 163: 217-229, 2003.

8. Eward KL, Obermann EC, Shreeram S, et al: DNA replication licensing in somatic and germ cells. J Cell Sci 117: 5875-5886, 2004.

9. Bravou V, Nishitani H, Song SY, Taraviras S and Varakis J: Expression of the licensing factors, Cdt1 and Geminin, in human colon cancer. Int J Oncol 27: 1511-1518, 2005.

10. Wharton SB, Hibberd S, Eward KL, et al: DNA replication licensing and cell cycle kinetics of oligodendroglial tumours. $\mathrm{Br}$ J Cancer 91: 262-269, 2004.

11. Montanari M, Boninsegna A, Faraglia B, Coco C, Giordano A, Cittadini A and Sgambato A: Increased expression of geminin stimulates the growth of mammary epithelial cells and is a frequent event in human tumors. J Cell Physiol 202: 215-222, 2005.

12. Xouri G, Lygerou Z, Nishitani H, Pachnis V, Nurse P and Taraviras $S$ : Cdt1 and geminin are down-regulated upon cell cycle exit and are over-expressed in cancer-derived cell lines. Eur J Biochem 271: 3368-3378, 2004. 
13. Shetty A, Loddo M, Fanshawe T, Prevost AT, Sainsbury R, Williams GH and Stoeber K: DNA replication licensing and cell cycle kinetics of normal and neoplastic breast. Br J Cancer 93: 1295-1300, 2005.

14. Gonzalez MA, Tachibana KE, Chin SF, et al: Geminin predicts adverse clinical outcome in breast cancer by reflecting cell-cycle progression. J Pathol 204: 121-130, 2004.

15. Dudderidge TJ, Stoeber K, Loddo M, et al: Mcm2, Geminin, and KI67 define proliferative state and are prognostic markers in renal cell carcinoma. Clin Cancer Res 11: 2510-2517, 2005.

16. Isola JJ, Helin HJ, Helle MJ and Kallioniemi OP: Evaluation of cell proliferation in breast carcinoma. Comparison of Ki-67 immunohistochemical study, DNA flow cytometric analysis and mitotic count. Cancer 65: 1180-1184, 1990.

17. Stoeber K, Tlsty TD, Happerfield L, et al: DNA replication licensing and human cell proliferation. J Cell Sci 114: 2027-2041, 2001.

18. Montanari M, Macaluso M, Cittadini A and Giordano A: Role of geminin: from normal control of DNA replication to cancer formation and progression? Cell Death Differ 13: 1052-1056, 2006.
19. Nishihara K, Shomori K, Fujioka S, et al: Minichromosome maintenance protein 7 in colorectal cancer: Implication of prognostic significance. Int J Oncol 33: 245-251, 2008.

20. Japanese Society for Cancer of the Colon and Rectum (eds): The General Rules for Clinical and Pathological Studies on Cancer of the Colon, Rectum and Anus. 7th edition. Kanehara \& Co., Ltd., Tokyo, pp21-34, 2006.

21. Kaplan EL and Meier P: Nonparametric estimation for incomplete observation. J Am Stat Assoc 53: 457-481, 1958

22. Cox DR: Regression models and life tables. J R Stat Soc B 34: 187-220, 1972.

23. Faraggi D and Simon R: A simulation study of cross-validation for selecting an optimal cutpoint in univariate survival analysis. Stat Med 15: 2203-2213, 1996.

24. Quaglia A, McStay M, Stoeber K, et al: Novel markers of cell kinetics to evaluate progression from cirrhosis to hepatocellular carcinoma. Liver Int 26: 424-432, 2006. 